\title{
Optically controlled 2D tunnelling in GaAs delta-doped $p$-n junction
}

\author{
S. A. Vitusevich ${ }^{1^{*}}$, A. Förster ${ }^{1}$, A. E. Belyaev ${ }^{2}$, B. A. Glavin ${ }^{2}$, K. M. Indlekofer ${ }^{1}$, H. Lüth ${ }^{1}$, R. V. Konakova ${ }^{2}$ \\ ${ }^{1}$ Institut für Schicht- und Ionentechnik, Forschungszentrum Jülich $\mathrm{GmbH}$ \\ D-52425 Jülich, Germany \\ ${ }^{2}$ Institute of Semiconductor Physics, National Academy of Sciences of Ukraine, Kyiv, 252028, Ukraine
}

\begin{abstract}
A new type of an optically controlled tunnelling process in a specially designed Esaki diode is investigated. The additional peak appears due to tunnelling of $2 \mathrm{D}$ electrons accumulated at ground state of delta doped layers embedded nearby the $p-n$ junction into the valence band of the $p+$-contact. It is found that the voltage position of an additional resonant peak shifts to lower bias voltage with increasing both incident light intensity and temperature. Our experimental data and theoretical simulations show that this shift is a result of an electrical field redistribution in the region of the $p$ - $n$ junction caused by non-equilibrium carriers generated with optic or thermal excitation.
\end{abstract}

Keywords: tunnelling diode, 2D electrons, delta-doping.

Paper received 10.03.99; revised manuscript received 21.03.99; accepted for publication 22.03.99.

\section{Introduction}

Inter band tunnelling in low-dimensional structures has become an important field of investigation [1-3]. The reason for this is an appearance of several regions possessing negative differential resistance that can be used for microwave and fast digital devices. Up to date, there is a very limited number of works devoted to the optically controlled tunnelling processes. The main reason for this is obviously connected with difficulty to create using light excitation a high concentration of photo-carriers with respect to the equilibrium value in heavy doped contact layers . At the same time the ability to optically control the electrical characteristics of tunnelling structures is an exciting prospect for the development of numerous opto-electronic devices. It is known that the quasi-twodimensional electronic system with two delta layers is very suitable for the design of new devices. The spatial extent of the delta layers potential can be comparable to the de Broglie wavelength of electrons, and the doping concentration can be a factor of two higher in the system that provides several subbands which can be populated [4]. The electron mobility in delta wells can be enhanced from two to five times over that of single well case [5]. The electron concentration and inter-subband transition energies can be changed considerably by an applied voltage bias [6,7]. Recently we have observed an additional resonant peak [8] in a specially designed Esaki diode, containing two delta layers in the region nearby the $p-n$ junction.

In this contribution we present results on the influence of optically and thermally excited carriers on the shape of the current-voltage $(I-V)$ characteristics of the structure. Our results proved that the origin of the additional peak differs from the main one and is a result of $2 \mathrm{D}$ electron tunnelling out of the ground state of the se two delta-layers .

* On leave from Institute of Semiconductor Physics, National Academy of Sciences of Ukraine, Kyiv, 252028, Ukraine. E-mail: s.vitusevich@fz-juelich.de 


\section{S. A. Vitusevich et al.: Optically controlled 2d tunnelling in GaAs ...}

\section{Experiment}

Measurements of electrical and optical parameters were conducted using specially designed $p$ - $n$ diode structures containing two delta doped layers. The structures were grown by MBE on semi-insulating GaAs (100) substrate. The sequence of epitaxial layers was the following: a highly doped $p+\left(p=10^{20} \mathrm{~cm}^{-3}\right)$ region is followed by a $5 \mathrm{~nm}$ highly doped $n^{+}$region $\left(n=10^{19} \mathrm{~cm}^{-3}\right)$ and two $5 \mathrm{~nm}$ separated delta layers $\left(n=10^{13} \mathrm{~cm}^{-2}\right)$, and finally a $3 \mathrm{~mm} n^{+}-$ layer. Mesa structures with a diameter of $10 \mathrm{~mm}$ were formed by photolithography and wet etching. The current-voltage characteristics were measured by a HewlettPackard 4145 semiconductor parameter analyser in a temperature range of $77-300 \mathrm{~K}$ under dark conditions. Optical measurements were performed at $77 \mathrm{~K}$, when the structure was immersed in liquid nitrogen. The structure was illuminated with different intensities of a tungsten lamp. In the paper we present the data obtained on devices $N 1$ and $N 2$ from series of diodes under study.

\section{Experimental results}

Most of the general peculiarities have been seen in all samples. The main of them is the appearance of one or two well resolved additional resonant peaks. In most cases the samples demonstrate one additional peak in the current-voltage characteristics observed at the excess valley current region. Sometimes in more perfect structures two additional peaks were observed. It should be mentioned that the voltage position of the additional peaks can change from sample to sample in the range from $0.4 \mathrm{~V}$ to $0.9 \mathrm{~V}$, but it is reproducible for each sample. Another feature observed in our experiments is the considerable effect of external conditions, namely, the influence of temperature as well as intensity of the incident light on the peaks' position and amplitude. Figure 1 shows typical I-V characteristics of the $p$ - $n$ diode $N 1$, measured under forward bias at room and at liquid nitrogen temperatures. The structure's peak current den-

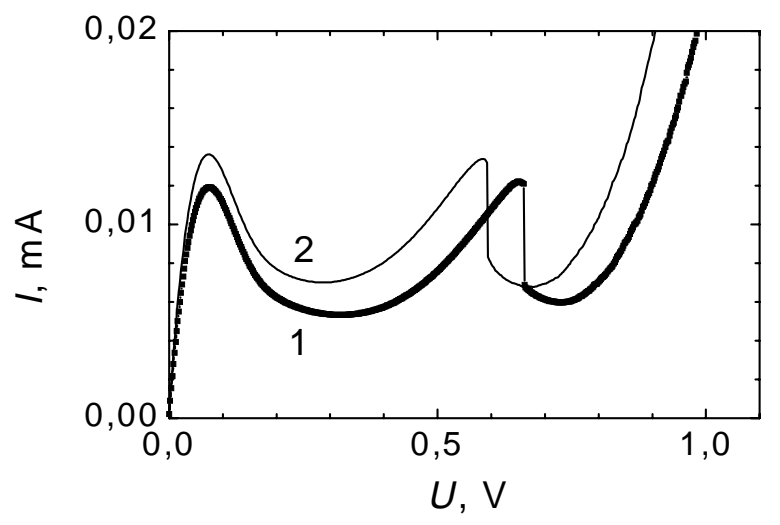

Fig. 1. $I-V$ characteristics of the $p-n$ diode $N 1$ measured at $77 \mathrm{~K}$ (curve 1) and $300 \mathrm{~K}$ (curve 2). sity is about $20 \mathrm{~A} \cdot \mathrm{cm}^{-2}$. The value is in good agreement with the doping level in our diode structure and very close to the value observed for the GaAs tunnelling diode with the same doping concentration in [9]. Peak $\left(I_{p}\right)$ and valley $\left(I_{v}\right)$ currents of both peaks increase with temperature growth. This trend is consistent with an expected temperature dependence for the GaAs band gap and with decreasing of the $p$ - $n$ junction's effective barrier thickness. At the same time, temperature dependencies of both the $I_{p} / I_{v}$ ratio (Fig. 2a) and the voltage position of the main and additional peak (Fig. 2b) are different. The additional peak voltage position shifts to lower bias with increasing temperature faster than the main one does. The peak-to-valley current ratio of the additional peak depends on the temperature very weakly in a wide temperature range. The $I-V$ curves plotted as a function of incident light intensity show in many respects changes that are similar to the temperature induced modifications of the $I-V$ characteristics shape. The $I-V$ curves recorded for $p$ - $n$ diode $N 2$ in the dark and at different light intensity are shown in Fig. 3. Magnitude of both tunnel currents corresponding to the main and additional peaks increases at illumination, and the density of photo-induced carriers is controlled by the light intensity. The
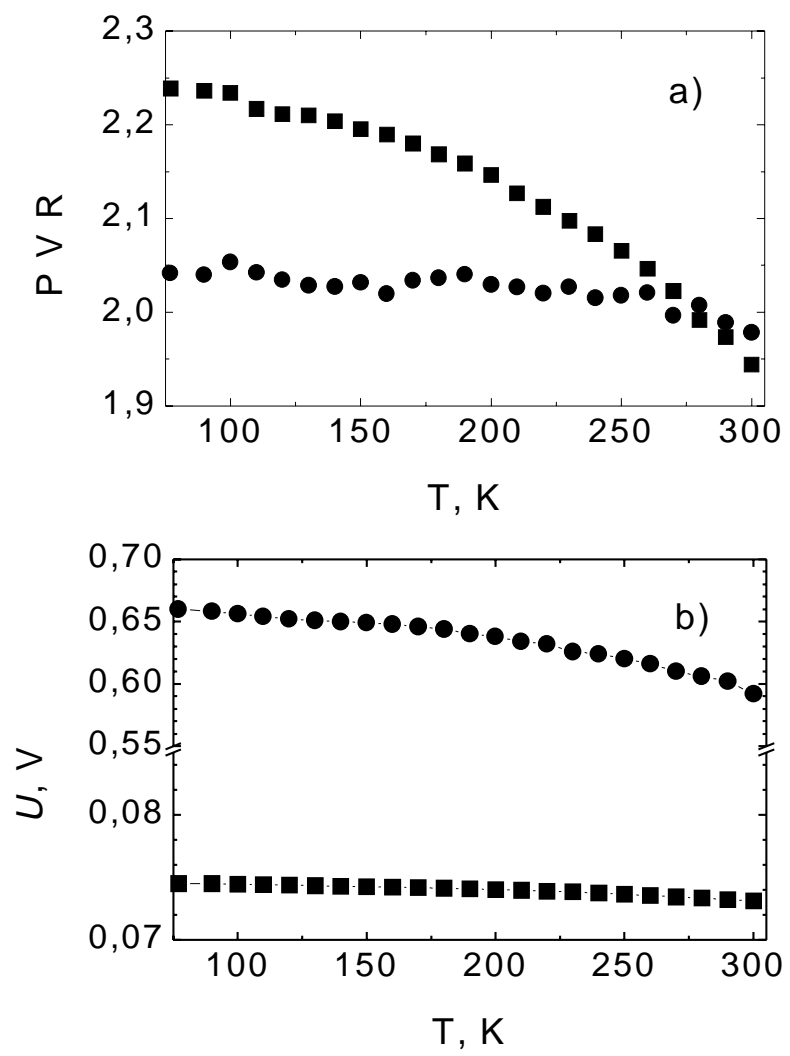

Fig. 2. Temperature dependence of the peak-to-valley current ratio (PVR) (a), and voltage position of the peaks (b) for the first peak (squares) and the additional peak (circles) of the diode $N 1$.

SQO, 2(1), 1999 


\section{S. A. Vitusevich et al.: Optically controlled $2 \mathrm{~d}$ tunnelling in GaAs ...}

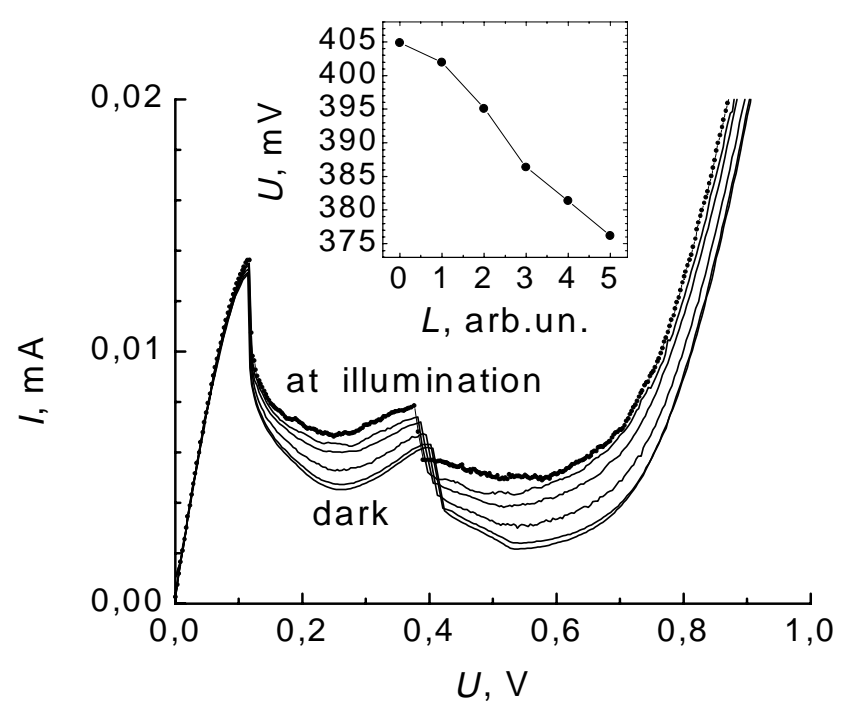

Fig. 3. $I-V$ characteristics of the $p-n$ diode $N 2$ with two delta layers measured at different light intensities. Curves from lower to upper correspond to the light intensity marked by points on insert. Insert: voltage position of the additional peak as a function of light intensity.

additional tunnelling peak shifts towards the lower voltage bias with increasing light intensity $L$ (insert to Fig. 3). The larger the illumination intensity the larger the shift voltage. At the same time, the main peak exhibits only small changes in the value of current and its voltage position.

\section{Theoretical model and discussion}

In order to determine the potential profile of the investigated structure and the energy levels of electron states we have employed a self-consistent Poisson and Schrödinger solver program for equilibrium conditions. The physical model is based on a density-functional theory where all single particle states have been split into two classes of quasi-free and 2D-bound states. For these two classes of states we can easily obtain expressions for exchange energies. The occupation probability of each level is given by a Fermi-Dirac distribution, whereby the statistics of the ionized impurities obey a slightly modified function. Within the simulation each layer of semiconductor material is described by an individual effective mass, doping and interface band offset referring to the adjacent region.

The calculated potential profile of the investigated structure at zero voltage bias and liquid nitrogen temperature is shown in Fig. 4. Both delta-layers are very close to the depletion region since the $p$ - $n$ junction width is of the order of $10 \mathrm{~nm}$. As a result the energy levels of bound electron states have to be very sensitive with respect to electric field redistribution under the influence of the external conditions. In fact, the voltage of the second peak shifts with increasing temperature and light intensity. Several calculated energy levels in the region of the two delta layers are obtained with a ground state energy of about $250 \mathrm{meV}$ below the Fermi level. Every time as a level is aligned with the energy range from quasi-Fermi level to the top of the valence band of the $p^{+}$-region tunnelling is possible and the current increases. If the tunnel barrier is thin enough, the total energy and the parallel momentum of the tunnelling electrons are conserved and

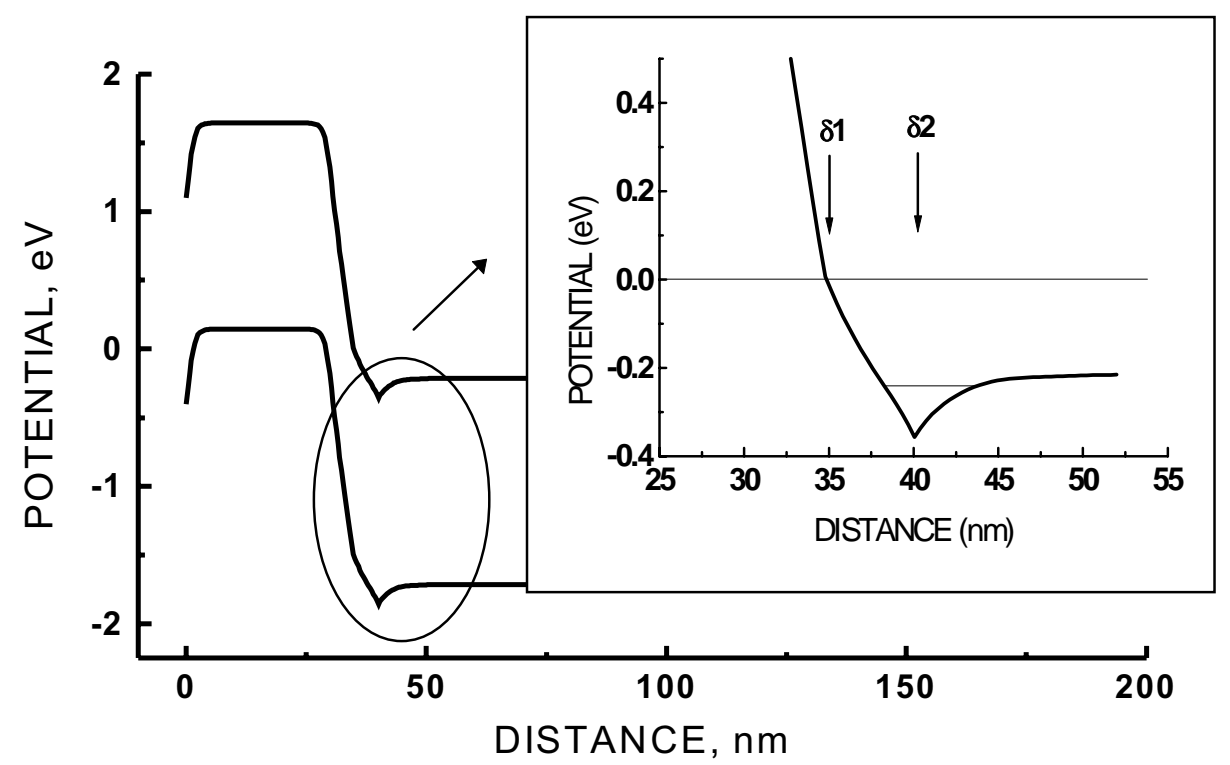

Fig. 4. The calculated band diagram of the $p-n$ junction with two delta-layers. 


\section{S. A. Vitusevich et al.: Optically controlled 2d tunnelling in GaAs ...}

electrons can tunnel resonantly from $3 \mathrm{D}$ to $2 \mathrm{D}$ electron systems and vice versa $[10,11]$. Really, sharp negative differential resistance features are observed in the $I-V$ characteristics, which confirm the quantum confined origin of electrons tunnel ling through the $p$ - $n$ junction. The distinction between the main and additional peak behaviour can be attributed to the different electric field distribution that affects tunnelling processes in the structure. The scaling factor that determines the relation between the energy shift and the applied voltage can be obtained as follows. We can obtain unambiguously the voltage range of the additional resonant peak using its start and end points. The start position of the additional resonant peak can be easily determined from the point at which the first derivative becomes positive after the negative differential region of the main peak. The position of the end is the voltage of the sharp current drop in the negative differential resistance region. Thus, at $77 \mathrm{~K}$ temperature the voltage range of the additional peak is $0.344 \mathrm{~V}$ for $p$ - $n$ diode $N 1$. This value equals to the Fermi energy of the $p$-type region (in our case $143 \mathrm{meV}$ ) added by $2 \mathrm{kT}$. The scaling factor is just the ratio of the values and is about 2.2. This means, that the voltage peak position $0.66 \mathrm{~V}$ corresponds to the energy shift of $300 \mathrm{meV}$. The voltage range of the additional peak appears to be a linear function of temperature. The higher the temperature the lower the observed range. The scaling factor is estimated in the same manner at room temperature and is about 1.56. The peak position at is temperature corresponds to an energy of $380 \mathrm{meV}$. For the $p$ - $n$ diode $N 2$ the energy is $380 \mathrm{meV}$ at the maximum intensity of illumination and $400 \mathrm{meV}$ in the dark. The difference in energies is a result of electric field redistribution stipulated by non-equilibrium carriers generated with thermal or optical excitation.

\section{Conclusions}

In summary, we have proposed and demonstrated a novel optically controlled inter band tunnelling diode. A well resolved additional resonant peak in the $I-V$ characteristics was found to shift to lower bias voltage with increasing temperature and intensity of optical excitation. This peak is due to tunnelling of localized electrons from the lowest subband that is formed by the effective confining potential of two delta-layers into the valence band of $p$ type region. A resonance occurs at voltages that correspond to the alignment of the energy level of the deltalayers ground state with an energy range from the quasiFermi level to the top of the valence band in the $p^{+}$-region. The peak's shift observed at illumination could be utilised in optical switching devices. It should be noted that the peak-to-valley current ratio and switching parameters are approximately the same at $300 \mathrm{~K}$ and $77 \mathrm{~K}$.

\section{Acknowledgments}

S. A. V. would like to acknowledge the Alexander von Humbold Foundation for financial support. A.E.B. and R.V.K. were supported by STCU under Grant No. 464.

\section{References}

1. A. Zakharova. Interband tunnelling in semiconductor heterostructures // Semicond. Sci. Techn., 13, p. 569 (1998).

2. M. Sweeny and J. Xu. Resonant interband tunnel diodes // Appl. Phys. Lett., 54, p. 546 (1989).

3. R. R. Marquardt, D. A. Collins, X. Y. Liu, D. Z.- Y. Ting and T. C. McGill. Resonant magnetotunneling spectroscopy of p-typewell interband tunneling diodes // Phys. Rev. , B53, p. 13624 (1996).

4. G.-Q. Hai and N. Studart. Electron mobility in two coupled d layers // Phys. Rev., B52, p. 11273 (1995).

5. X. Zheng, T. K. Carns, K. L. Wang and B. Xu. Electron mobility enhancement from coupled wells in delta-doped GaAs // Appl. Phys. Lett., 62, p. 504 (1993).

6. O. A. Mezrin, A. Y. Shik and V. O. Merzin. Gated d-layer structures // Semicond. Sci. Techn. , 7, p. 664 (1992).

7. T. W. Kim. Effect of an applied electric field on electronic subbands in $\mathrm{In}_{0.53} \mathrm{Ga}_{0.47} \mathrm{As} / \mathrm{In}_{0.52} \mathrm{Al}_{0.48}$ As double quntum wells // Sol. St. Comm., 104, p. 495 (1997).

8. S. A. Vitusevich, A. Foerster, A. E. Belyaev, K. M. Indlekofer, H. Luth and R. V. Konakova. Resonant tunneling effect in delta doped p-n GaAs junction // J. Micr. Eng. (1999) (to be published).

9. L. Beji, B. el Jani, P. Gibart, J. C. Portal and P. Basmaji. Hydrostatic pressure studies of GaAs tunnel diodes //J. Appl. Phys. , 83, p. 5573 (1998).

10. D. J. BenDaniel and C. B. Duke. Conductance anomalies due to space-charge-induced localized states // Phys. Rev., 160, p. 679 (1967).

11. W. Demmerle, J. Smoliner, G. Berthold, E. Gornik and G. Weiman. Tunneling spectroscopy in barrier-separated two-dimensional electron-gas systems // Phys. Rev. , B44, p. 3090 (1991). 